\title{
Robust efficient global optimisation via adaptive surrogate refinement
}

\author{
Noémie Le Carrer ${ }^{1, *}$, David Moens ${ }^{2}$, and Matthias Faes ${ }^{2}$ \\ ${ }^{1}$ Institute for Risk and Uncertainty, University of Liverpool, Liverpool, UK \\ ${ }^{2}$ KU Leuven, Leuven, Belgium
}

\begin{abstract}
Through the combination of a given surrogate model (e.g. Kriging) and a procedure allowing to compute the local optima of a given black-box function (e.g. tuned Particle swarm optimization), our algorithm produces refined estimates of the global optima of a multidimensional black-box model. A confidence ellipsoid computed in a Bayesian way is associated to each estimate, and shown to be reliable. The setting is tested on the 2-dimensional Branin function. Results outperform those given by two state-of-the-art non-parallelised efficient global optimisation methods.
\end{abstract}

Copyright line will be provided by the publisher

\section{Related works \& Contributions}

Efficient global optimisation (EGO) was introduced 20 years ago in the seminal paper [1] to perform global optimisation from a very limited computational budget. It is motivated by widespread engineering applications such as design optimisation, when the models to be optimised are particularly computationally intensive (e.g. a crash test simulation running for $O(24 h)$ ). Jones and co-authors combine surrogate models, providing an approximation $\hat{f}(x)$ in any point $x$ of the search domain $S$ of a function $f(x)$ from a limited sample set $\left(x^{i}, f\left(x^{i}\right)\right), i=1, \ldots, n$, to a global search, through an adaptive sampling of the points to be computed exactly over the optimisation procedure. An intermediary objective function $c$ is maximised at each iteration by the optimal next design point (DP). The infill criterion $c(x)$ is both function of the surrogate prediction $\hat{f}(x)$ and the prediction error distribution $p_{e}$ in $x$. Because of its structure, Gaussian process regression models the prediction error as a normal distribution [3]. It is consequently a convenient and commonly-used choice for EGO. The most popular infill criteria, expected improvement and probability of improvement [2], are sometimes combined in a multicriteria optimisation of $c$ [5]. A global infill criterion is developed in [4], which performs a stepwise uncertainty reduction of the optimisers distribution. Parallel computing enabled the use of ensembles or mixtures of surrogates, through e.g. Dempster-Shafer theory [6], as well as the development of routines for multiple point selections in a Bayesian framework [7,8]. Alternative approaches were also introduced in the context of e.g., the efficient computation of failure probabilities and robust design optimization [10].

The existing EGO methods listed above provide point estimates of the global optimum of $f$. Their performances are characterized by both the number of DP and the error between estimate and true optimum. However, when facing an unknown black-box model, uncertainty quantification is needed to safely propagate EGO estimates as inputs in further computations. Here, we present an enhanced version of EGO addressing this issue. We compare it to state-of-the-art non-parallelised solutions. Our contribution is two-fold: (1) Improving the performance of non-parallelised adaptative design; and (2) Producing an uncertainty quantification of the result, i.e. providing a confidence index over an area supposed to contain each true optimum.

\section{Methodological development}

In the following, R-EGO designates our algorithm. Assume $S \in \mathbb{R}^{d}$ and $f$ is to be minimised. First set the initial number $N_{k}$ of DP and the maximum number of additional points $N$, as well as the order of the regression polynomials, the correlation function type and its parameters. In the following, all these choices are summarised by the parameter vector $\Omega$ and denote $K(X, \Omega)$ the surrogate model (here Kriging) associated with the DP set $X$ and parameter set $\Omega$.

Initialisation Sample $N_{k}$ points $x^{j} \in S, j=\left\{1, \ldots, N_{k}\right\}$ by means of a space-filling procedure, e.g. latin hypercube sampling. The set of DP is from now on referred to as $X_{i}$. $i$ (so far set to 0 ) will keep track of the number of design points added to $X_{0}$ along the procedure. Compute the first Kriging model $K_{i}=K\left(X_{i}, \Omega\right)$.

Iterative search Each iteration starts by locating the set $C_{i}$ of local minima of the response surface $f_{i}(x)$, estimation of $f(x)$ by means of the surrogate $K_{i}$, in the search space $S$. Throughout the algorithm, we use a tuned version of the particle swarm optimisation heuristic for performing both global and local optimisations. For each of the $l$ local minima $x^{k} \in C_{i}$, we apply the following procedure.

We roughly estimate the bounds on the future landscape of the surrogate $K_{i+1}=K\left(X_{i+1}, \Omega\right)$ if $x^{k}$ was used alone as next DP. In other words, we fictitiously update the surrogate $K_{i}=K\left(X_{i}, \Omega\right)$ by adding the DP $\left(x^{k}, f_{i}\left(x^{k}\right)+a \sigma_{i}\left(x^{k}\right)\right)$ and get $\tilde{K}_{i+1}^{k, a}$, with $a=\{-1,0,1\}$. In order to avoid over-optimistic Kriging variances, all standard deviations $\sigma_{i}$ are computed by means of leave-one-out bootstrapping [9]. This choice allows as well the use of alternative surrogate models to Kriging. We then find the global minima associated with the response surface $\tilde{K}_{i+1}^{k, a}$ over $S$ and store it in $\tilde{C}_{i}$.

\footnotetext{
* Corresponding author: e-mail nlc@liverpool.ac.uk
} 
Table 1: Euclidean distance between final estimates $x_{i}^{*}$ (after 15 / 35 iterations) and the corresponding exact minimum location. Statistics (minimum, median (bold), 80th percentile, maximum) computed over 40 runs. The same statistics are computed for the semi major axis of the confidence ellipsoid associated with each best estimate, used as uncertainty quantification (UQ).

\begin{tabular}{lccc}
\hline Method & $x_{1}$ & $x_{2}$ & $x_{3}$ \\
\hline R-EGO & $0.02-\mathbf{0 . 7 1 - 2 . 7 2 - 3 . 2 9 / 0 . 0 0 4 - 0 . 1 2 - 0 . 2 6 - 0 . 8 8}$ & $0.01-\mathbf{0 . 0 9}-0.19-2.20 / 0.01-\mathbf{0 . 0 6}-0.09-0.11$ & $0.007-\mathbf{0 . 1 7}-0.61-1.16 / 0.02-\mathbf{0 . 1 1}-0.21-0.88$ \\
\hline R-EGO UQ & $0.03-\mathbf{0 . 2 3}-0.59-2.9 / 0.09-\mathbf{0 . 2 0}-0.22-0.46$ & $0.08-\mathbf{0 . 1 4}-0.39-3.9 / 0.02-\mathbf{0 . 1 3}-0.38-0.78$ & $0.03-\mathbf{0 . 2 5}-0.39-5.2 / 0.03-\mathbf{0 . 2 5}-0.38-2.04$ \\
\hline EGO & $3.22 / 3.22$ & $2.40 / 2.40$ & $0.04 / 0.04$ \\
\hline IAGO & $2.18 / 0.23$ & $0.44 / 0.18$ & $0.82 / 0.23$ \\
\hline
\end{tabular}

Finally the element of $\tilde{C}_{i}$ that has lowest $f_{i}(x)$ and is not too close to the members of $X_{i}$ (the threshold is set to $\frac{\epsilon l_{d}}{i+1}$, with $\epsilon=0.1$ and $l_{d}$ the diagonal length of the bounding box associated with $\left.S_{d}\right)$ is selected as next DP. If no candidate satisfies these conditions, the new DP is selected at random in the space $S_{d}$. The Kriging model is updated to $K_{i+1}=K\left(X_{i+1}, \Omega\right)$ and the $n_{k}$ local minima of the corresponding response surface over $S,\left(x_{i}^{p s o, k}, f_{i}\left(x_{i}^{p s o, k}\right)\right), k=\left\{1, \ldots, n_{k}\right\}$, are returned as estimates of the $f$-minima. Note that $S_{d}=S$ initially. However, when all elements of $C_{i}$ and their $\sigma$-bounds conduce to landscapes whose minimum are the same or too close to existing DP, the pool of candidates $\tilde{C}_{i}$ turns empty. In this case, $S_{d}$ becomes at $i+1$ the bounding box centred in the minimum $x_{i}^{*}$ of $f_{i}(x)$ with mid-edge equals to the distance between $x_{i}^{*}$ and its closest neighbour. Thus the algorithm recursively allows DP-candidates closer to existing DP/previous minimum estimates until the user is satisfied with the precision of the result or the computational budget is consumed.

Uncertainty quantification A Gaussian mixture model $\Sigma_{k=1}^{n} w_{i}^{k} N\left(\mu_{i}^{k}, \Sigma_{i}^{k}\right)$ is finally fitted to the equivalent pdf of the Kriging response surface at iteration $i$. The number of components $n$ is either found by clustering the $i-1$ added DP via $\mathrm{k}$-means, or by user-knowledge of the shape of the function to optimise. This allows, at stage $i+1$, to update the location of the $k$ best estimates $x_{i+1, k}^{p s o}$ in a Bayesian framework. To that purpose, the covariance matrix $\Sigma_{i+1}$ of a Gaussian distribution fitting the equivalent pdf of $K_{i+1}$ is computed. The prior knowledge $\Sigma_{k=1}^{n} w_{i}^{k} N\left(\mu_{i}^{k}, \Sigma_{i}^{k}\right)$ on the mean location $\mu^{k}$ of the $k$-th minimum is updated in a Bayesian way through the new evidence $x_{i+1}^{p s o}$. The posterior distribution $\sum_{k=1}^{n} w_{i+1}^{k} N\left(\mu_{i+1}^{k}, \sum_{i+1}^{k}\right)$ provides centers $\left\{\mu_{i+1}^{k}\right\}$ and covariance matrices that are used to compute the smallest confidence ellipsoids $E_{\alpha}^{k}$ and their confidence index $\alpha$ to which each of the best estimate $x_{i+1}^{p s o, k}$ belongs.

\section{Benchmark results \& Conclusions}

To compare to state-of-the-art non-parallelised procedures, we reproduce the setting described in [4]. The Branin function has three global minimisers, $x_{1} \sim(-3.14,12.27), x_{2} \sim(-3.14,2.27)$ and $x_{3} \sim(9.42,2.47)$, with a global minimum $f * \sim 0.4$. $N_{k}=16$ points are uniformly spread over the search space initially. In [4], the authors present the results of the application of the standard EI-based EGO algorithm [1] and their informational approach to global optimisation (IAGO) to the problem at hand, after 15 and 35 iterations. We present our results next to theirs in Table 1. Results show that the approach presented here both reaches the precision of the local EGO approach w.r.t. the best resolved minimum, and outperforms the two existing methods in terms of global resolution, being able to locate much more precisely the auxiliary minima (cumulated error has minimum 0.23 and 80th quantile 3.07 vs 5.66 and 3.44 for EGO and IAGO respectively for 15 iterations, and minimum 0.14 , 80th percentile 0.48 for 35 iterations against 3.44 and 0.64 for the two other procedures). Our approach being stochastic, the best estimates for the minima have to be accompanied by reliable uncertainty bars. Reliability plots show that the uncertainty quantification performed is reliable whatever the confidence index, i.e. the confidence areas contain the true solution more often that the confidence index suggests. Which leads to the conclusion that these confidence areas, whose typical dimensions are provided in Table 1, could be tightened around the best estimates by revising our Bayesian updating framework.

Acknowledgements N. Le Carrer is funded by the EPSRC and the ESRC with grant no. EP/L015927/1. M. Faes is a post-doctoral researcher of the Research Foundation Flanders (grant number 12P3519N).

\section{References}

[1] D. R. Jones, M. Schonlau, and W. J. Welch, Journal of Global Optimization 13, 455-492 (1998).

[2] D. R. Jones, Journal of Global Optimization 13, 345-383 (2001).

[3] J. Sacks, W. J. Welch, and T. J. Mitchell, and H. P. Wynn, Statistical Science ., 409-423 (1989).

[4] J. Villemonteix, E. Vasquez, and E. Walter, Journal of Global Optimization 44, 509 (2009).

[5] H. Wang, M. Emmerich, and T. Bäck, 2016 IEEE Congress on Evolutionary Computation (CEC) (IEEE, 2016), pp. 719-727. (2016).

[6] J. Müller, R. Piché, Journal of Global Optimization 51, 79-104 (2011).

[7] J. Wang, C. Scott, and E. Liu, and P. I. Frazier, arXiv preprint, arXiv:1602.05149 (2016).

[8] D. Ginsbourger, R. Le Riche, and L. Carraro, Computational Intelligence in Expensive Optimization Problems 2, 131-162 (2010).

[9] D. Den Hertog, J. P. C. Kleijnen, and A. Siem, Journal of the Operational Research Society 57, 400-409 (2006).

[10] V. Dubourg, B. Sudret, and J. M. Bourinet, Structural Multidisciplinary Optimisation 44, 673--690 (2011). 